\section{Stroke, vascular risk factors and depression}

\section{Cross-sectional study in a UK Caribbean-born population}

R. STEWART, M. PRINCE, M. RICHARDS, C. BRAYNE and A. MANN

\begin{abstract}
Background Stroke, hypertension and diabetes are common in older Caribbean-born populations in the UK who may be at risk of depression secondary to vascular disease.
\end{abstract}

Aims We examined the association between stroke, vascular risk factors and depression in a community-based Caribbean-born population aged 55-75 years.

\section{Method Vascular risk factors were identified by interview, examination and blood tests. Depression was categorised using the Geriatric Depression Scale. Disablement was assessed as a potential mediating factor.}

Results Physical illness and disablement were strongly associated with depression, independent of disablement. Previous stroke was associated with depression, independent of disablement. No vascular risk factors were associated with depression.

\section{Conclusions The risk of depression} associated with stroke was not explained by disablement. However, the hypothesis that vascular risk factors are important in the genesis of depression was not supported.

\section{Declaration of interest This study} was funded by a grant from the Wellcome Trust. R.S. is supported by a Research Training Fellowship from the Wellcome Trust.
Vascular disease is hypothesised to cause depression in later life (Hickie \& Scott, 1998). However, the relationship, at a population level, between depression and common risk factors for vascular disease such as hypertension, diabetes and hyperlipidaemia is less clear - in particular, whether this explains, or may be explained by, well-recognised associations between depression and disablement (Prince et al, 1998). Older Caribbean-born people may represent a population in the UK particularly at risk for vascular-mediated depression because increased rates of stroke have been reported in these communities (Stewart et al, 1999) and hypertension and diabetes are both more prevalent and more severe (Chaturvedi et al, 1993; Raleigh et al, 1996). A community study has been carried out recently in south London, primarily to investigate vascular cognitive impairment in an older Caribbean-born population. Using data gathered during this study the association between vascular risk factors and depression was investigated.

\section{METHOD}

\section{Study populations}

Potential participants for this study were identified using registration lists from seven primary care teams in south London. Primary care practice staff were asked to identify the ethnicity of all registered patients within the inclusion age range and those identified as African-Caribbean were approached to take part. This method of sampling had been used in previous studies of this ethnic group (Chaturvedi $e t$ al, 1993), and its validity was investigated by attempting to contact patients of unknown ethnicity within one practice. For this practice there were 1031 registered patients within the inclusion age range: 127 were identified as potentially eligible and 504 were identified as not AfricanCaribbean, leaving 400 whose ethnicity was unknown. Of 60 randomly selected persons from this latter group, 6 were found to be potentially eligible for the study, 3 of whom declined to be contacted. It could therefore be estimated that the unsampled population for this practice might contain a further 40 potentially eligible persons and therefore that the sampling frame covered approximately $75 \%$ of the total eligible population.

Entry criteria for the main sample were confirmed at interview: all participants in the study were aged $55-75$ years, were born on a Caribbean island (or in Guyana), had at least one parent who was born in a Caribbean nation and were living in community accommodation at the time of assessment. There were no specific exclusion criteria.

\section{Measurements}

Participants were assessed at home by research staff. The following measures are considered in this analysis:

(a) Demographic information: age; educational level (school leaving age); occupational social class (highest attained).

(b) Information on vascular disease/risk: self-reported diagnoses of (and treatment for) stroke, hypertension and diabetes; Rose questionnaire for symptoms of angina (Rose et al, 1982); structured questionnaire for smoking (Cox et al, 1993) and physical activity (Pols et al, 1997).

(c) Disablement: four measures of overall physical morbidity and disablement were used. The number of self-reported health problems was calculated from a possible list of eight. In addition, participants were asked how severely they were affected by these problems on a three-point scale and the sum of these scores was used as a measure of impairment. Disability was measured by a structured questionnaire enquiring about difficulties in 12 activities of daily living (Prince et al, 1997). Handicap was measured using four sections of the London Handicap Scale (Harwood \& Ebrahim, 1995), assessing (on a six-point scale) levels of disadvantage in mobility, physical independence, occupation and orientation. These measures were all used previously in the Gospel Oak study of a community-based elderly population (Prince et al, 1997). 
(d) Examination for vascular disease/risk: resting blood pressure (taken with an automatic sphygmomanometer (Foster et al, 1994) and the lowest of two consecutive seated readings is used); resting electrocardiogram (ECG; Minnesota coding protocol; Prineas et al, 1982); reagent strip (Multistix) urinalysis for protein and glucose; height, weight, waist and hip measurements.

(e) Blood tests (non-fasting; agreed to by $90 \%$ of participants): total, highdensity lipoprotein (HDL) - and lowdensity lipoprotein (LDL)-cholesterol; triglycerides; fibrinogen.

(f) Psychiatric measures: 10-item Geriatric Depression Scale (GDS-10; D'Ath et al, 1994); Mini-Mental State Examination (MMSE, 30-point; Folstein et al, 1975) with cognitive impairment defined as a score of 20 or below the lower than standard cut-off takes into account potential cross-cultural differences in MMSE distribution (Gurland et al, 1992); previous consultation with any doctor for psychiatric complaints; age of first consultation.

In a separate study the 15 -item version of the GDS had been validated in AfricanCaribbean primary care attenders of a similar age range against the Geriatric Mental State GMS-AGECAT standardised psychiatric diagnosis (Abas et al, 1998). Using data made available, GDS-10 scores were derived for participants in that study and sensitivity/specificity calculated against the other scales. A 3/4 cut-off on the GDS10 scale represented an optimal balance between sensitivity and specificity $(0.84$ and 0.76 , respectively) for 'pure' depression (of mild to moderate severity) according to GMS-AGECAT criteria and this cut-off was therefore used to categorise depression in the study considered here.

\section{Statistical analysis}

Primary hypotheses were that vascular risk factors would be associated with depression independent of measures of disability and handicap and that the association between measures of physical impairment, disability and handicap would be explained at least partly by increased vascular disease/risk in more dependent groups.

STATA 6.0 for Windows software was used. Depression (according to the GDS-10 cut-off described above) was treated as the principal dependent variable. Univariate analyses were initially used to examine associations between depression and all measurements of vascular disease/risk $\left(\chi^{2}\right.$ and two-tailed $t$-tests, as appropriate). For the measures of disablement, scores were continuously distributed but the degree of positive skew precluded transformation. These were subdivided a priori in order to create approximately equal group sizes, and odds ratios for depression were calculated across the distribution. Secondary, stratified analyses were carried out to investigate differences in associations within subgroups of age, gender, disablement and past psychiatric history. Logistic regression analysis was used to investigate the role of vascular disease in the association between depression and disablement, and to investigate confounding/mediating effects of disablement in associations between depression and categorical vascular measures (with $P$ values derived from likelihood ratio tests).

\section{RESULTS}

\section{Sampling procedure}

Of 749 addresses of potential participants provided by the seven practices, a reply was obtained from 708, 65 did not fulfil criteria for ethnicity and 156 were not known or no longer resident at the address given. Of the remaining 487 potentially eligible, $185(38 \%)$ declined to participate and data were incomplete for a further 15 $(3 \%)$. Results were therefore analysed on 287 participants. Examination of those who declined to take part showed no differences in age or gender distribution compared with those who participated. Those with incorrect addresses had a similar mean age but were more likely to be male $(61 \%)$. There was no substantial association between depression and refusal to have a blood test: odds ratio $(\mathrm{OR})=1.43$ and $95 \%$ confidence interval $(\mathrm{CI})=0.58-3.55$.

\section{Characteristics of the study population}

The sample was evenly distributed in terms of age and gender, 54 (19\%) were categorised as depressed according to a $3 /$ 4 cut-off on the GDS-10; $160(58 \%)$ were receiving treatment for hypertension and $82(29 \%)$ for diabetes; 51 (18\%) had hypertension with proteinuria and/or ECG hypertrophy; and $44(16 \%)$ had diabetes with glycosuria and/or haemoglobin A1c levels of $8.2 \%$ or above. Mean levels of cholesterol $(5.6 \mathrm{mmol} / \mathrm{l})$ and triglycerides (1.5 mmol/l), however, were lower than would be expected in a UK Caucasian population. Current smoking was relatively uncommon $(25 \%)$ and in current smokers, the levels of smoking were relatively low. Seventeen $(6 \%)$ gave a history of stroke and the median reported time since last stroke was 10 years (interquartile range 3-20 years); 13 (5\%) had ECG evidence of infarction and $112(40 \%)$ had evidence of ischaemia. A previous general practitioner (GP) consultation for a psychiatric problem was reported by $46.3 \%$ of subjects with depression and $13.6 \%$ of those without depression. The MMSE scores were 20 or below for $47(16 \%)$ participants.

\section{Demographic factors and disablement}

Univariate associations between depression, demographic factors and disablement are presented in Table 1 . No substantial associations were seen with age, gender, educational level or occupational social class. Strongly significant associations were seen, however, between depression and all measures of disablement.

\section{Vascular disorders/vascular risk}

Characteristics of depression/non-depression groups with respect to measures of vascular disease/risk are presented in Table 2. Depression was positively associated with a previous history of stroke. However, there were no associations evident for any other factors. Secondary analyses carried out stratifying for age, gender, disability, presence/absence of a previous consultation for psychiatric problems and age at first consultation did not reveal any evidence for obscured associations within subgroups. In addition, there were no increased rates of depression in groups with more severe hypertension or diabetes (as defined above).

\section{Multivariate analyses}

The association between depression and previous stroke was examined with age, gender, number of diagnoses and the three measures of disablement in a logistic regression model. The strength of the association was reduced after adjustment but remained significant $(\mathrm{OR}=3.44,95 \%$ CI $1.09-10.9$, $P=0.034)$. Further adjustment for cognitive 
Table I Univariate associations between demographic factors, measures of disablement and depression

\begin{tabular}{|c|c|c|c|c|c|}
\hline & & Number & $\begin{array}{c}\% \text { with } \\
\text { depression }\end{array}$ & $\chi^{2}$-test ( $P$ value $)$ & Odds ratio $(95 \% \mathrm{Cl})$ \\
\hline \multirow[t]{4}{*}{ Age (years) } & $55-60$ & 70 & 22.9 & 0.41 & \\
\hline & $6 I-65$ & 69 & 14.5 & & \\
\hline & $66-70$ & 90 & 15.9 & & \\
\hline & $71-75$ & 60 & 23.3 & & \\
\hline \multirow[t]{2}{*}{ Gender } & Male & 126 & 15.2 & 0.17 & 1.00 \\
\hline & Female & 164 & 21.6 & & $\mathrm{I} .54(0.83-2.85)$ \\
\hline \multirow[t]{3}{*}{ School leaving age (years) } & $<15$ & 75 & 17.6 & 0.87 & \\
\hline & $15-16$ & 163 & 20.4 & & \\
\hline & $>16$ & 39 & 18.4 & & \\
\hline \multirow[t]{3}{*}{ Occupational class } & $1 / 2 / 3 n$ & 91 & 22.2 & 0.33 & \\
\hline & $3 m$ & 105 & 14.4 & & \\
\hline & $4 / 5$ & 93 & 20.7 & & \\
\hline \multirow[t]{2}{*}{ Number of health problems } & $0-2$ & 124 & 10.6 & 0.005 & 1.00 \\
\hline & $3-8$ & 145 & 23.8 & & $2.64(1.31-5.33)$ \\
\hline \multirow[t]{4}{*}{ Physical impairment score } & $0-1$ & 60 & 3.3 & $<0.0001$ & 1.00 \\
\hline & $2-3$ & 77 & 10.5 & (trend) & $3.41(0.68-17.1)$ \\
\hline & $4-5$ & 66 & 23.1 & & $8.70(1.76-43.0)$ \\
\hline & $6-18$ & 65 & 33.9 & & $14.8(2.90-76.0)$ \\
\hline \multirow[t]{3}{*}{ Physical disability score } & 0 & 170 & 10.0 & $<0.0001$ & 1.00 \\
\hline & $\mathrm{I}-4$ & 66 & 24.6 & (trend) & $2.94(1.36-6.35)$ \\
\hline & $5-18$ & 54 & 40.4 & & $6.10(2.75-13.5)$ \\
\hline \multirow[t]{3}{*}{ Handicap score } & 0 & 140 & 8.6 & $<0.0001$ & 1.00 \\
\hline & $\mathrm{I}-3$ & 76 & 22.4 & (trend) & $3.07(1.36-6.96)$ \\
\hline & $4-13$ & 73 & 35.2 & & $5.80(2.57-13.1)$ \\
\hline
\end{tabular}

impairment made no substantial difference $(\mathrm{OR}=3.45)$. Adjustment for age, gender, previous stroke and principal vascular risk factors (hypertension, diabetes, ECG ischaemia, cholesterol and triglyceride levels) made little difference to the associations between measures of disablement and depression. For example, the odds ratio for depression across the groups of disability scores displayed in Table 1 was 2.48 (95\% CI 1.72-3.59) unadjusted and $3.04 \quad(95 \%$ CI $1.92-4.81)$ after adjustments.

\section{DISCUSSION}

A significant association between previous stroke and current depression was found in this population, independent of measures of disablement. No such associations were seen, however, between depression and other measures of vascular disease or risk. Potential sources of bias require consideration before drawing conclusions from these largely negative findings.

\section{Study limitations}

The most important considerations in interpreting these findings relate to the representativeness of participants compared to the source population. Various selection processes may have operated in the sampling procedure, which involved recalled ethnicity by practice staff, and, from results within one practice, it can be estimated that the sampling frame represented approximately $75 \%$ of the source population. Unfortunately, due to time and staffing restraints, it was not feasible to contact all persons of unknown ethnicity for all practices because only $10 \%$ of these were potentially eligible for the study. Refusal rates, in addition, were relatively high but comparable with another community-based study of a similar population (Chaturvedi et al, 1993). It is important to consider how selection processes might have affected results and, in particular, whether they would have obscured an association between depression and vascular risk factors. It is likely that higher levels of physical morbidity would be represented in individuals who were approached to take part compared with the source population (because they were known to practice staff), but the extent and direction of bias are unknown with respect to depression. However, prevalence rates of mild to moderate depression in community samples of older people have been estimated as being between 10 and $20 \%$ (Beekman et al, 1999), and the prevalence of an equivalent level of disease severity in this study is within this range. This suggests that over- or underrepresentation of depression in this study is at least not substantially different from other community surveys. The fact that it is towards the upper end of this range suggests that selection bias would, if anything, exaggerate an association between depression and vascular risk factors. An additional consideration is that observed associations of depression with measures of disablement were comparable in strength to findings from other community studies (Prince $e t$ $a l, 1997)$. Any bias obscuring an association between depression and physical health would therefore have to be specific to vascular disorders, which is an unlikely situation.

If depression was associated with increased rates of mortality or institutionalisation of people with vascular disease, then associations might be underestimated or missed in this cross-sectional, communitybased study. However, the only vascular condition associated with depression was stroke, which would be expected to be one of the most vulnerable to prevalence bias. A shorter duration of depressive episodes in people with vascular disease would be unlikely because vascular disease has, if anything, been reported to be associated with a more prolonged duration of depression (Hickie \& Scott, 1998).

A further issue to consider in the light of negative findings is that of statistical power. This study was designed primarily to investigate the association between vascular risk disorders (such as hypertension and diabetes) and cognitive impairment. Negative findings for less-prevalent cardiovascular disorders such as myocardial infarction should be treated with caution because this study would lack the power to detect associations. Possible sources of measurement error (which would reduce the strength of associations) particularly include the less-than-perfect sensitivity/ specificity of the depression screen, the use of self-reported diagnoses, the measurement 
Table 2 Characteristics of depression and non-depression groups with respect to vascular disease and risk factor status

\begin{tabular}{|c|c|c|c|c|c|c|}
\hline & \multicolumn{2}{|c|}{ Mean (s.d.) } & \multicolumn{2}{|c|}{ Number (\%) } & \multirow[t]{2}{*}{ Odds ratio $(95 \% \mathrm{Cl})$} & \multirow[t]{2}{*}{$P$} \\
\hline & Depression & Non-depression & Depression $(n=54)$ & Non-depression $(n=233)$ & & \\
\hline Previous stroke & & & $9(17)$ & II (5) & $4.03(1.55-10.5)$ & 0.002 \\
\hline Symptoms of angina & & & $7(13)$ & $34(15)$ & $0.87(0.36-2.09)$ & 0.76 \\
\hline ECG infarction & & & $3(6)$ & $10(4)$ & $1.31(0.35-4.95)$ & 0.69 \\
\hline ECG ischaemia & & & $18(33)$ & $96(4 I)$ & 0.71 (0.38-I.33) & 0.29 \\
\hline Hypertension & & & $32(59)$ & $133(57)$ & $1.09(0.60-2.00)$ & 0.77 \\
\hline Diabetes & & & $16(30)$ & $65(28)$ & $1.09(0.57-2.09)$ & 0.80 \\
\hline Current smoking & & & $16(30)$ & $56(24)$ & $1.33(0.69-2.57)$ & 0.39 \\
\hline Systolic BP (mmHg) & $150(25)$ & $150(24)$ & & & & 0.92 \\
\hline Diastolic BP (mmHg) & $84(13)$ & $86(12)$ & & & & 0.29 \\
\hline Cholesterol (mmol/l) & $5.5(1.1)$ & $5.6(1.2)$ & & & & 0.50 \\
\hline $\mathrm{HDL}(\mathrm{mmol} / \mathrm{l})$ & $1.4(0.4)$ & $1.4(0.4)$ & & & & 0.97 \\
\hline $\mathrm{LDL}(\mathrm{mmol} / \mathrm{l})$ & $3.4(1.0)$ & $3.4(1.0)$ & & & & 0.76 \\
\hline Triglycerides (mmol/l) & $1.6(0.1)$ & $\mathrm{I} .7(0.9)$ & & & & 0.48 \\
\hline Fibrinogen $(\mathrm{mg} / \mathrm{dl})$ & $345(130)$ & $339(120)$ & & & & 0.76 \\
\hline Body mass index & $29.6(6.0)$ & $29.9(4.8)$ & & & & 0.73 \\
\hline Waist/hip ratio & $0.92(0.08)$ & $0.93(0.11)$ & & & & 0.43 \\
\hline
\end{tabular}

ECG, electrocardiogram; BP, blood pressure; HDL, high-density lipoprotein; LDL, low-density lipoprotein.

of blood pressure at a single time point and the non-fasting nature of lipid levels. However, upper confidence intervals of odds ratios calculated for the associations between depression and more common disorders such as hypertension or diabetes were small compared with the effects of general physical morbidity and do not suggest the substantial effects that were missed.

\section{Implications for the 'vascular depression' hypothesis}

A significant association was found between stroke and depression in this study. Although this was a single finding in the context of multiple analyses and may therefore represent type 1 error, it is consistent with other research reporting raised rates of depression after stroke (Pohjasvaara et al, 1998). There is disagreement as to whether increased rates of major depression within the first few months following stroke are mediated through pathways of cerebral damage or psychological impact (Gainotti et al, 1999). Our results suggest that risk of mild to moderate levels of depression remains raised over a considerably longer period following stroke in this population and that this is not fully explained by effects on function. Other possible pathways would include the effect of cerebral damage but may also involve psychological factors specific to the diagnosis of stroke. In view of the long period that had elapsed since the last stroke in most cases, mediating processes can be assumed to be chronic and may predominantly affect duration rather than incidence of depression.

Despite well-recognised associations between stroke and depression and the increased frequency of magnetic resonance imaging white matter hyperintensities (of suspected ischaemic origin) in older persons with depression (O’Brien et al, 1996), there has been little evidence that vascular risk factors are an important cause of depression at a population level. Two large studies reported no association between blood pressure and depressive symptoms (Friedman \& Bennet, 1977; Jones-Webb et al, 1996) and depression is associated with, if anything, low rather than raised lipid levels (Horsten et al, 1997). Increased levels of depression have been described in association with diabetes but it has been suggested that these may largely be accounted for by worse overall physical health and function (Rajala et al, 1997). Many of the risk factors for vascular disease were associated with relative cognitive impairment in this study (results submitted; details available from R.S. upon request), suggesting at least some degree of cerebral effect in at-risk groups. However, there was no evidence to suggest that this degree of effect was associated with depression at a population level in the absence of clinical stroke. The generalisability of results from this study requires consideration:

(a) The age range for subjects was relatively young and it is possible that an association between vascular risk and depression is restricted to older age groups. However, there was no indication of any changing effect across the age range examined in this study.

(b) Time-dependent associations may be missed in a cross-sectional study. Lowered blood pressure and/or lipid levels as a consequence of depression may obscure an association with higher premorbid values. This effect is observed for cognitive decline where raised blood pressure predicts cognitive impairment 15 years later but where cognitive impairment is cross-sectionally associated with lower blood pressure (Stewart, 1999). However, a recent prospective study has suggested that low blood pressure precedes, rather than is secondary to, depression (Paterniti et al, 2000).

(c) Studies of increased white matter hyperintensities in older subjects with depression have predominantly used out-patient or in-patient cases. An association with vascular risk may therefore exist for a relatively small subgroup of patients with depression 
that is particularly severe, of long duration or is resistant to treatment. This study did not have sufficient power to examine risk factors for more severe forms of depression and, in addition, was limited in the extent to which age of onset and duration of depression could be assessed.

(d) Results from this particular population may not be generalisable to other ethnic groups. Although it is possible that biological mechanisms of vascular cerebral damage may be specific to particular ethnic groups, psychosocial issues are more likely to affect generalisability in this respect. Of the vascular risk disorders considered, epidemiological evidence for an association with depression is strongest for type 2 diabetes. It is possible that absent associations in this study may reflect a different subjective meaning of particular diagnoses in this population. Diabetes and hypertension in particular were observed during the process of data collection to have a high profile in this community. Those diagnosed may experience less feelings of isolation and receive more informal support as a result, although this study was unable formally to investigate this issue.

An alternative explanation relates to the direction of causality between vascular disease and depression. The association between depression and white matter hyperintensities on imaging has been established from cross-sectional studies. Depression in younger adults, however, has been demonstrated in large prospective studies to be a risk factor for future vascular disease (Wassertheil-Smoller et al, 1996; Penninx et al, 1998). If white matter abnormalities are a consequence rather than a cause of depression (or if behavioural or personality traits underlie both conditions), this would explain a lack of association between depression and other risk factors for cerebrovascular disease.

\section{Public health implications}

Poor physical health is well established as a risk factor for depression in later life. From a public health perspective, the nature of potential interventions to reduce depression associated with poor health will depend on how this association is mediated. Effects through cerebrovascular disease and white matter damage would suggest that interventions should focus on

\section{CLINICAL IMPLICATIONS}

- Increased disablement was strongly associated with mild to moderate levels of depression in this population.

An association between previous stroke and depression was observed that was not explained by disablement.

- There were no associations between any risk factors for vascular disease and mild to moderate levels of depression.

\section{LIMITATIONS}

- Depression was categorised according to a cut-off on a 10 -point screening scale.

- Non-participation rates were relatively high and the sample is likely to have a higher level of physical morbidity than the source population.

- Information was limited concerning age of onset and duration of depressive symptoms and there was insufficient power to investigate more severe forms of depression.

R. STEWART, MRCPsych, M. PRINCE, MD, A. MANN, MD, Section of Old Age Psychiatry, Institute of Psychiatry London; M. RICHARDS, PhD, Department of Epidemiology and Public Health, University College London; C. BRAYNE, MD, Department of Public Health and Primary Care, Institute of Public Health, University of Cambridge

Correspondence: Dr R. Stewart, Section of Old Age Psychiatry, Institute of Psychiatry, De Crespigny Park, Denmark Hill, London SE5 8AF, UK. Tel: 0207848 0240; fax: 02077010167

(First received 25 February 2000, final revision 23 June 2000, accepted 28 June 2000)

more rigorous identification and control of vascular risk disorders. However, effects through the impact of disablement and impaired function would suggest that more appropriate interventions would be to minimise disablement associated with physical ill health or to improve psychological adjustment to disablement. Our findings with respect to stroke suggest a relatively small but independent impact on depression at a population level compared with disablement, which requires further investigation. However, with respect to more commonly occurring conditions, the evidence from both this and other studies is that, for mild to moderate levels of depression, it is the personal impact of impaired function that is most important rather than condition-specific effects.

\section{ACKNOWLEDGEMENTS}

We are grateful to practitioners and staff from the seven primary care teams who participated in this study and to research nurses Ginie Moutou and Vivienne Maskrey who assisted in data collection. Cut-off points for the GDS-10 in this population were derived using data kindly made available by Dr Melanie Abas.

\section{REFERENCES}

Abas, M. A., Phillips, C., Carter, J., et al (1998)

Culturally sensitive validation of screening

questionnaires for depression in older African-

Caribbean people living in south London. British Journal of Psychiatry, 173, 249-254.

Beekman, A.T. F., Copeland, J. R. M. \& Prince, M. J. (1999) Review of community prevalence of depression in later life. British Journal of Psychiatry, 174, 307-3II.

Chaturvedi, N., McKeigue, P. M. \& Marmot, M. G. (1993) Resting and ambulatory blood pressure differences in Afro-Caribbeans and Europeans. Hypertension, 22, 90-96.

Cox, B. D., Huppert, F. A. \& Wichelow, M. J. (1993) The Health and Lifestyle Survey: Seven Years On. Aldershot: Dartmouth Publishing.

D’Ath, P., Katona, P., Mullan, E., et al (1994) Screening, detection and management of depression in elderly primary care attenders. I: The acceptability and performance of the 15 item Geriatric Depression Scale 
(GDSI5) and the development of short versions. Family Practice, II, 260-266.

Folstein, M., Folstein, S. \& McHugh, P. (1975) 'MiniMental State', a practical method for grading the cognitive state of patients for the clinician. Journal of Psychiatric Research, 12, 189-198.

Foster, C., McKinlay, S., Cruickshank, J. M., et al (1994) Accuracy of the Omron HEM 706 portable monitor for home measurement of blood pressure. Journal of Human Hypertension, 8, 66I-664.

Friedman, M. J. \& Bennet, P. L. (1977) Depression and hypertension. Psychosomatic Medicine, 39, 134-142.

Gainotti, G., Azzoni, A. \& Marra, C. (1999) Frequency, phenomenology and anatomical-clinical correlates of major post-stroke depression. British Journal of Psychiatry, 175, 163-167.

Gurland, B. J., Wilder, D. E., Cross, P., et al (1992) Screening scales for dementia: toward reconciliation of conflicting cross-cultural findings. International Journal of Geriatric Psychiatry, 7, 105-113.

Harwood, R. H. \& Ebrahim, S. (1995) Manual of the London Handicap Scale. Nottingham: University of Nottingham.

Hickie, I. \& Scott, E. (1998) Late-onset depressive disorders: a preventable variant of cerebrovascular disease? Psychological Medicine, 28, 1007-1013.

Horsten, M., Wamala, S. P., Vingerhoets, A., et al (1997) Depressive symptoms, social support, and lipid profile in healthy middle-aged women. Psychosomatic Medicine, 59, 52I-528.
Jones-Webb, R., Jacobs, D. J., Flack, J. M., et al (1996) Relationships between depressive symptoms, anxiety, alcohol consumption, and blood pressure: results from the CARDIA Study. Alcohol Clinical and Experimental Research, 20, 420-427.

O'Brien, J.T., Ames, D. \& Schwietzer, I. (1996) White matter changes in depression and Alzheimer's disease: a review of magnetic resonance imaging studies. International Journal of Geriatric Psychiatry, II, 68I-694.

Paterniti, S., Verdier-Taillefer, M.-H., Geneste, C., et al (2000) Low blood pressure and risk of depression in the elderly. A prospective community-based study. British Journal of Psychiatry, 176, 464-467.

Penninx, B.W., Guralnik, J. M., Mendes, D. L., et a (1998) Cardiovascular events and mortality in newly and chronically depressed persons $>70$ years of age. American Journal of Cardiology, 81, 988-994.

Pohjasvaara, T., Leppavuori, A., Siira, L., et al (1998) Frequency and clinical determinants of poststroke depression. Stroke, 29, 23II-2317.

Pols, M. A., Peeters, P. H. M., Ocke, M. C., et a (1997) Estimation of reproducibility and relative validity of the questions included in the EPIC physical activity questionnaire. International Journal of Epidemiology, $\mathbf{2 6}$ (suppl. I), SI8I-SI89.

Prince, M. J., Harwood, R. H., Blizard, R. A., et al (1997) Impairment, disability and handicap as risk factors for depression in old age. The Gospel Oak Project V. Psychological Medicine, 27, 31I-321.
_, _ , Thomas, A., et al (1998) A prospective population-based cohort study of the effects of disablement and social milieu on the onset and maintenance of late-life depression. The Gospek Oak Project VII. Psychological Medicine, 28, 337-350.

Prineas, R. J., Crow, R. S. \& Blackburn, H. (1982) The Minnesota Code Manual of Electrocardiographic Findings. Bristol: John Wright \& Sons.

Rajala, U., Keinanen-Kiukaanniemi, S. \& Kivela, S. L. (1997) Non-insulin-dependent diabetes mellitus and depression in a middle-aged Finnish population. Social Psychiatry and Psychiatric Epidemiology, 32, 363-367.

Raleigh, V. N., Kiri, V. \& Balarajan, R. (1996) Variations in mortality from diabetes mellitus, hypertension and renal disease in England and Wales by country of birth. Health Trends, 28, 122-127.

Rose, G., Blackburn, H., Gillum, R., et al (1982) Cardiovascular Survey Methods. Geneva: WHO.

Stewart, J. A., Dundas, R., Howard, R. S., et a (1999) Ethnic differences in incidence of stroke: prospective study with stroke register. British Medical Journal, 318, 967-971.

Stewart, R. (1999) Hypertension and cognitive decline. British Journal of Psychiatry, 174, 286-287.

Wassertheil-Smoller, S., Applegate, W. B., Berge, K., et al (1996) Change in depression as a precursor of cardiovascular events. SHEP Cooperative Research Group (Systolic Hypertension in the Elderly). Archives of Internal Medicine, 156, 553-561. 